\title{
Lung ultrasound is useful in oncohematologic patients with respiratory dysfunction admitted to an Intensive Care Unit (ICU): a pilot study
}

\author{
Cecilia Gómez Ravetti1,2,3, Thiago Bragança Lana Silveira Ataíde ${ }^{1,3}$, Lídia Miranda Barreto ${ }^{1,3}$, \\ Fabricio de Lima Bastos ${ }^{3}$, Angélica Gomide dos Reis Gomes ${ }^{3}$, Renan Detoffol Bragança ${ }^{1,3}$, \\ Carolina Coimbra Marinho ${ }^{1,2,3}$, Vandack Nobre ${ }^{1,2,3}$
}

${ }^{1}$ Intensive Care Unit, Hospital das Clínicas of Universidade Federal de Minas Gerais, ${ }^{2}$ Department of Internal Medicine, School of Medicine, Universidade Federal de Minas Gerais, ${ }^{3}$ NIIMI (Interdisciplinary Group for Intensive Care Investigation), Belo Horizonte, Minas Gerais, Brazil

\begin{abstract}
Aims: This pilot study aimed to evaluate the usefulness of a sequential lung ultrasound score (LUS) in immunosuppressed patients with oncohematologic diseases and acute respiratory dysfunction hospitalized in an intensive care unit (ICU). Materials and methods: LUS was calculated at ICU admission, after $24 \mathrm{~h}, 48 \mathrm{~h}$ and at discharge. A score ranging from 0 to 26 was attributed according to the number of B lines, presence of lung consolidation and pleural effusion. Results: Twenty-six patients were included. The median age was 50 years [interquartile range (IQR) 21] and 14 (54\%) were male. LUS on the day of ICU admission was significantly higher in non-survivors compared to survivors (13 [5] vs 9 [9], respectively; $\mathrm{p}=0.047$ ). The median delta LUS (LUS_D2 - LUS_D1) did not show difference between survivors and non-survivors (2 [0-7.5] vs 1 [-1.5 - 5], $\mathrm{p}=0.33$ ). Among patients initially submitted to noninvasive mechanical ventilation (NIMV), no difference in LUS at inclusion or after $24 \mathrm{~h}$ was found between those who succeeded or failed on this support. Conclusion: The use of LUS to quantify lung aeration loss in oncohematologic patients hospitalized in an ICU due to acute respiratory dysfunction might be a helpful tool to predict the severity of the illness.
\end{abstract}

Keywords: lung ultrasound; oncohematologic; mechanical ventilation

\section{Introduction}

The use of ultrasound (US) in intensive care units (ICU) has proved to be of great utility to manage critically ill patients, primarily in those with respiratory dysfunction, in whom this tool presents high sensitivity and specificity to identify the mechanisms of respiratory dysfunction $[1,2]$.

Acute respiratory dysfunction (ARD) is the main reason for the admission of oncohematologic patients to the

Received 12.01.2020 Accepted 01.03.2020

Med Ultrason

2020, Vol. 22, No 2, 178-182

Corresponding author: Cecilia Gómez Ravetti

190 Av Professor Alfredo Balena,

Santa Efigênia, Belo Horizonte, Brasil CEP: 30130-100

E-mail: ceciliag.ravetti@gmail.com
ICU. Invasive mechanical ventilation (IMV) requirement in this population of patients is associated with higher mortality rates, reaching $90 \%$ in some studies $[3,4]$. In this context, non-invasive mechanical ventilation (NIMV) to avoid intubation appears as an attractive practice, with growing use throughout the last years [5]. NIMV might be associated with a reduction in ICU and hospital mortality rate, but outcomes are worse in patients that require subsequent invasive mechanical ventilation $[6,7]$. Finally, the overuse of NIMV in immunocompromised patients has been proved to be harmful $[8,9]$.

The main causes of ARD in immunosuppressed patients are immunosuppression-related infections, pulmonary involvement by the malignancy itself or chemotherapy-related toxicity. In general, the first imagine exam at admission is thorax radiography. The computed tomography is the goal standard, but often unstable patients with 
ARD are not able to be transported to radiology department [10].

This pilot study aimed to evaluate the utility of a lung ultrasound score (LUS) in a group of immunosuppressed patients with oncohematologic diseases admitted to an ICU with ARD. We hypothesized that LUS could aid the assistant physicians in the decisions concerning the kind of respiratory assistance. Moreover, we sought to determine potential associations between the LUS and relevant clinical outcomes.

\section{Materials and methods}

This is a prospective, observational pilot study realized on critically ill oncohematologic patients with ARD admitted in ICU between February 2017 to February 2018.

We included adults $(\geq 18$ years) patients previous diagnosed with hematologic malignancy in which the respiratory dysfunction began within the last 48 hours prior to admission in ICU. The respiratory dysfunction was defined by the presence of at least two of the following conditions: respiratory rate $\geq 32 / \mathrm{min}$, arterial oxygen saturation $<94 \%$ at room air and $\mathrm{P} / \mathrm{F}$ ratio $\left(\mathrm{PaO}_{2} / \mathrm{FiO}_{2}\right)$ $<300 \mathrm{mmHg}$. The patients with allogeneic bone marrow transplantation were excluded.

All included patients agreed to participate in this study and signed an Informed Consent Form (ICF). The local Research Ethics Committee (CAEE: 37297314.5.0000.5149) approved this research.

\section{Lung US protocol}

Sequential lung US exams were performed in all patients, at admission (D1), after 24h (D2) and 48h of ICU stay and at ICU discharge. Four lung windows were evaluated in each hemithorax: $2^{\text {nd }}$ intercostal space (ICS) in the midclavicular line, $5^{\text {th }}$ ICS in the anterior axillary line, costophrenic region and posterior axillary line right above the diaphragm (posterolateral alveolar and/ or pleural syndrome - PLAPS [11]. Aeration loss was quantified in each lung window from 0 to 3 , according to previously described protocols with an increase of one point if pleural effusion was present [12]. LUS calculation is depicted in Table I.

Demographic, clinical, and laboratory data were also collected. The severity of diseases was quantified through the SOFA [13], APACHE II [14] and SAPS3 [15] scores.

US exams were performed independently by two examiners (TBLA, CGR). At least ten videos per exam were performed. The first 182 videos referent to the first ten patients included in the study were compared for interobserver agreement. A good agreement was found with Kappa's correlation coefficient value of $0.799(\mathrm{p}<0.001)$.
Table I. Lung ultrasound score adapted from Soummer et al [12]

\begin{tabular}{ll}
\hline Finding & Points assigned \\
\hline Pattern of A lines with lung sliding & 0 points \\
More than 3 well-defined B lines & 1 point \\
Coalescent B lines & 2 points \\
Lung consolidation & 3 points \\
$\begin{array}{l}\text { Additional point if pleural effusion was } \\
\text { present }\end{array}$ & 1 point
\end{tabular}

Score range: 0 to 26 . Four lung windows in each hemithorax: 2 nd intercostal space in the midclavicular line, 5thintercostal space in the anterior axillary line, costophrenic region, and posterior axillary line right above the diaphragm.

\section{Statistical analysis}

Data were collected and analyzed using SPSS 22.0 program. Qualitative variables were described as absolute and relative frequencies. Quantitative variables were described as median and interquartile range (IQR) due to skewed distribution in the Kolmogorov-Smirnov test. Mann-Whitney U, Kruskal Wallis and Wilcoxon tests were used to compare medians. Chi-square and Fisher exact tests were used to compare the qualitative variables. Statistical significance was taken as $\mathrm{p}<0.05$.

\section{Results}

Twenty-six patients were included. Patients' main characteristics are shown in Table II. The median age was 50 years (IQR 21), and 14 (54\%) patients were male. Acute myeloid leukemia (38\%), lymphoma (27\%), and multiple myeloma $(11 \%)$ were the most frequent hematologic diagnosis. The main reasons for ICU admission were febrile neutropenia and nosocomial pneumonia. Thirteen $(50 \%)$ patients required vasopressors use. The median (IQR) ICU length of stay was 5.5 (5.5) days.

Considering the first modality of respiratory support, $21(80 \%)$ patients received NIMV, three were intubated at admission and two patients received only supplementary oxygen therapy. Fourteen out of $21(66 \%)$ patients initially treated with NIMV were eventually intubated and submitted to invasive mechanical ventilation. The ICU mortality was different between the four subgroups; none of the patients in the exclusive $\mathrm{O}_{2}$ therapy and NIMV success groups died, while $57.1 \%$ and $100 \%$ died in the NIMV failure and IMV group, respectively ( $<<0.01$.

\section{Lung Ultrasound Scores}

Comparison of LUS at admission and after $24 \mathrm{~h}$ between the three initial subgroups of patients (oxygen therapy only, NIMV and IMV) did not show a significant difference (Table III).

LUS on the day of ICU admission was significantly higher in non-survivors compared to survivors (13 [5] vs 
Table II. Patients' characteristics

\begin{tabular}{ll}
\hline Characteristics & All (n=26) \\
\hline Age, years & $50(21)$ \\
Sex male & $14(53.8)$ \\
Hematologic disease & \\
$\quad$ AML & $10(38)$ \\
$\quad$ Other Leukemias & $4(15)$ \\
$\quad$ Lymphoma & $7(27)$ \\
$\quad$ Multiple Myeloma & $3(11)$ \\
Others & $2(7)$ \\
Main ARD diagnosis & \\
Febrile Neutropenia & $14(53)$ \\
$\quad$ Nosocomial Pneumonia & $4(15)$ \\
CHF & $2(7)$ \\
Others & $6(23)$ \\
Vasopressors use T0 & $13(50)$ \\
PaO2/FiO2 & $224(169)$ \\
BNP, pg/mL & $8020(19710)$ \\
ICU LOS, days & $5.5(5.5)$ \\
LOS before ICU admission & $16(27)$ \\
APACHE II & $20(7.3)$ \\
SOFA admission & $7(3.3)$ \\
SAPS 3 & $63(15)$ \\
ICU mortality & $11(42.3)$ \\
\hline
\end{tabular}

The results are expressed as number (\%) or median (IQR). CHF: Cardiac Heart Failure; ICU: Intensive Care Unit; LOS: Length of Stay; BNP: B-type Natriuretic Peptide; ARD: Acute Respiratory Dysfunction

Table III. Comparison between the 3 groups of the study $\left(\mathrm{O}_{2}\right.$ supplementary, NIMV and IMV).

\begin{tabular}{lllll}
\hline Patients & $\begin{array}{l}\text { O2 only } \\
(\mathbf{n}=\mathbf{2})\end{array}$ & $\begin{array}{l}\text { NIMV } \\
(\mathbf{n = 2 1})\end{array}$ & $\begin{array}{l}\text { IMV } \\
(\mathbf{n}=\mathbf{3})\end{array}$ & $\begin{array}{l}\mathbf{p} \\
\text { value }^{*}\end{array}$ \\
\hline $\begin{array}{l}\text { LUS_D1, } \\
\text { n=26 }\end{array}$ & $8(5-11)$ & $12(0-23)$ & $13(13-16)$ & 0.36 \\
$\begin{array}{l}\text { LUS_D2, } \\
\text { n=23 }\end{array}$ & $11(10-12)$ & $14.5(7-24)$ & $14(14-14)$ & 0.48 \\
$\begin{array}{l}\text { ICU } \\
\text { mortality }\end{array}$ & 0 & $8(38.1)$ & $3(100)$ & $<0.01$ \\
\hline
\end{tabular}

The results are expressed as median (minimum-maximum) or as number $(\%)$ * Kruskal Wallis test.

LUS: lung ultrasound score; D1: ICU admission; D2: after 12 to 24 hours; ICU: Intensive Care Unit; NIMV: noninvasive mechanical ventilation; IMV: invasive mechanical ventilation.

9 [9], respectively; $\mathrm{p}=0.047$ ). The median variation of LUS (delta LUS = LUS D2 - LUS D1) did not show significant difference between the same groups (2 [0-7.5] vs $1[-1.5-5], \mathrm{p}=0.33)$.

\section{Patients initially treated with NIMV}

In patients that received NIMV as the initial respiratory support, no difference in LUS measured either at inclusion or after $24 \mathrm{~h}$ was found between those with good (progressive improvement) and poor (orotracheal intu- bation) response, despite the higher mortality observed in the poor response group (Table IV). Of note, NIMV non-responders had significantly higher SOFA scores as compared to successful cases (7 [3] vs 4 [3], p<0.01).

Patients with good responses to NIMV showed a significant increment of median LUS from admission to 24 $\mathrm{h}(9$ [10] vs. 14 [5], $\mathrm{p}=0.042)$, different from those who failed to NIMV where the difference was not significant.

\section{Discussion}

In this pilot study, a score based on the lung US findings in oncohematologic patients with ARD hospitalized in an ICU proved to be higher at admission in those with an unfavorable outcome during the ICU stay. However, the LUS at admission were not significantly different between the three groups of initial respiratory support $\left(\mathrm{O}_{2}\right.$, NIMV and IMV) and the scores values did not distinguish between patients with potential poor or good response to NIMV. Moreover, our findings suggest that the lung aeration changes identified by US at ICU admission and sequentially after $24 \mathrm{~h}$ can be useful to determine the effects of positive pressure ventilation.

Previous studies have investigated the meaning of lung aeration loss measured with LUS (e.g., by the quantification of B lines, consolidations and pleural effusion) in critically ill patients $[12,16,17]$. LUS showed to be useful to determine the best positive-end expiratory pressure (PEEP) before and after application of recruitment maneuvers in patients with acute respiratory distress syndrome [17]. Dynamic changes in the extent of consolidation measured with ultrasound in patients with ventilation-associated pneumonia can be helpful to quantify the response to antibiotic treatment [16]. Finally, LUS has been shown to accurately predict the success of extu-

Table IV. Comparison between success or failure to noninvasive mechanical ventilation.

\begin{tabular}{llll}
\hline Patients & $\begin{array}{l}\text { NIMV success } \\
(\mathbf{n}=7)\end{array}$ & $\begin{array}{l}\text { NIMV failure } \\
(\mathbf{n}=14)\end{array}$ & p value* \\
\hline $\begin{array}{l}\text { LUS-D1, } \\
\mathrm{n}=21\end{array}$ & $9(10)$ & $12.5(9.2)$ & 0.73 \\
$\begin{array}{l}\text { LUS-D2 } \\
\mathrm{n}=20\end{array}$ & $14(5)$ & $16(9)$ & 0.75 \\
$\begin{array}{l}\text { Difference } \\
\text { LUSD2-LUSD1, }\end{array}$ & $2(0-9)$ & $1(-1-6)$ & 0.63 \\
$\begin{array}{l}\text { ICU Mortality } \\
\text { ICU }\end{array}$ & $8(57.1)$ & 0.018 \\
\hline
\end{tabular}

The results are expressed as median (interquartile range) or as number (\%). * Mann-Whitney U test.

LUS: lung ultrasound score; D1: ICU admission; D2: after 12 to 24 hours. LUSD1-LUSD2: difference between LUS-D2 and LUSD1; ICU: Intensive Care Unit; NIMV: noninvasive mechanical ventilation. 
bation following the protocol of weaning of mechanical ventilation [12].

In agreement with the above-cited evidence, in this study, we noted that the LUS was influenced by two main variables: severity of the disease at admission and the type of ventilatory support administered. The disease itself determined the first ultrasound findings before the administration of any ventilatory intervention (NIMV or IMV). Therefore, patients who died in ICU showed higher initial ultrasound scores (i.e., poorly aerated lungs) than those who survived. We hypothesize that the first exam quantified the magnitude of the aeration loss before the application of any ventilatory support. After that, the LUS are affected by the treatment offered.

The treatment administered after admission determined the second ultrasonography findings. Conversely to our expectations, patients who respond to NIMV showed significant augment in the scores, probably because the lower and shorter positive pressure offered by this ventilatory support. In contrast, patients who failed on NIMV and were eventually intubated showed no significant increment in the scores, partially because under IMV, these patients underwent a prolonged and higher positive pressure.

Yin et al retrospectively analyzed 175 patients with different types of shock and showed that the LUS at admission was an independent risk factor for 28-day mortality [18]. Differently, our study was conducted prospectively and included a subgroup of patients with specific characteristics.

Lu et al also evaluated pulmonary evolution with ultrasound in patients with acute respiratory distress syndrome (ARDS) undergoing extracorporeal membrane oxygenation (ECMO). At inclusion all patients were on invasive mechanical ventilation and no difference was found in LUS between survivors and non-survivors. After ECMO, those who survived had a decrease in lung scores [19]. Our hypothesis is that LUS is a good marker of pulmonary aeration improvement when the patient is under continuous positive pressure, as shown in other studies $[16,17,20]$.

Definition of the best timing for orotracheal intubation in oncohematologic patients is a challenge. The use of LUS could be of help, even though clinical judgment remains the main criterion to guide this decision. An additional benefit of LUS is its accessibility (easy to do and quick), noninvasive, does not involve the emission of radiation and allows sequential evaluation [1]. In this study, the lung score follow-up during the first days of ICU stay was useful to demonstrate the response to the ventilatory support administered. It was also useful to identify patients with predicted worse outcomes at ad- mission by the quantification of aeration loss in oncohematologic patients.

\section{Limitations}

This study was carried out in a single medical center, with a small sample of patients. However, this was a pilot study, delineated for the generation of hypotheses, and to prove the concept of LUS usefulness to evaluate oncohematologic patients with respiratory dysfunction. Although our sample size did not have the power to investigate the association between LUS and hard endpoints (i.e., mortality or the need of orotracheal intubation), it was satisfactory to evaluate the behavior of lung aeration loss measured by ultrasound in oncohematologic patients with ARD.

Our study failed to show LUS improvements in survivors as expected, probably because mortality in those who were submitted to IMV or failed NIMV was high (100\% and $66 \%$ respectively), precluding comparisons of the subgroups.

We believe our findings will be helpful in the hypothesis-generating process and planning of future studies in this field. In this regard, larger - ideally multicenter studies should be conducted in an attempt to confirm the present results. If confirmed, the benefits of LUS can be tested in future clinical trials.

In conclusion the use of LUS to quantify lung aeration loss in oncohematologic patients hospitalized in an ICU due to acute respiratory dysfunction might be a useful tool to predict the severity of illness. More controlled and randomized studies, with a larger number of patients, are warranted to refine the use of LUS further.

\section{Conflict of interest: none}

\section{References}

1. Lichtenstein DA, Mezière GA. Relevance of lung ultrasound in the diagnosis of acute respiratory failure: the BLUE protocol. Chest 2008;134:117-125.

2. Liccardo B, Martone F, Trambaiolo P, Severino S, Cibinel GA, D'Andrea A. Incremental value of thoracic ultrasound in intensive care units: Indications, uses, and applications. World J Radiol 2016;8:460-471.

3. Grasselli G. ICU admission of patients with hematological malignancies: the debate continues. Minerva Anestesiol 2013;79:113-115.

4. Azoulay E, Thiéry G, Chevret S, et al. The prognosis of acute respiratory failure in critically ill cancer patients. Medicine (Baltimore) 2004;83:360-370.

5. Azoulay E, Lemiale V, Mokart D, et al. Acute respiratory distress syndrome in patients with malignancies. Intensive Care Med 2014;40:1106-1114.

6. Hilbert G, Gruson D, Vargas F, et al. Noninvasive ventilation in immunosuppressed patients with pulmonary infil- 
trates, fever, and acute respiratory failure. N Engl J Med 2001;344:481-487.

7. Antonelli M, Conti G, Bufi M, et al. Noninvasive ventilation for treatment of acute respiratory failure in patients undergoing solid organ transplantation: a randomized trial. JAMA 2000;283:235-241.

8. Squadrone V, Massaia M, Bruno B, et al. Early CPAP prevents evolution of acute lung injury in patients with hematologic malignancy. Intensive Care Med 2010;36:1666-1674.

9. Lemiale V, Mokart D, Resche-Rigon M, et al. Effect of Noninvasive Ventilation vs Oxygen Therapy on Mortality Among Immunocompromised Patients With Acute Respiratory Failure: A Randomized Clinical Trial. JAMA 2015;314:1711-1719.

10. Lecuyer L, Chevret S, Thiery G, Darmon M, Schlemmer B, Azoulay E. The ICU trial: a new admission policy for cancer patients requiring mechanical ventilation. Crit Care Med 2007;35:808-814.

11. Lichtenstein D, Mézière G, Biderman $P$, Gepner A, Barré O. The comet-tail artifact. An ultrasound sign of alveolar-interstitial syndrome. Am J Respir Crit Care Med 1997; 156:1640-1646.

12. Soummer A, Perbet S, Brisson H, et al. Ultrasound assessment of lung aeration loss during a successful weaning trial predicts postextubation distress*. Crit Care Med 2012;40:2064-2072.

13. Vincent JL, Moreno R, Takala J, et al. The SOFA (Sepsisrelated Organ Failure Assessment) score to describe organ dysfunction/failure. On behalf of the Working Group on
Sepsis-Related Problems of the European Society of Intensive Care Medicine. Intensive Care Med 1996;22:707-710.

14. Knaus WA, Draper EA, Wagner DP, Zimmerman JE. APACHE II: a severity of disease classification system. Crit Care Med 1985;13:818-829.

15. Metnitz PG, Moreno RP, Almeida E, et al. SAPS 3--From evaluation of the patient to evaluation of the intensive care unit. Part 1: Objectives, methods and cohort description. Intensive Care Med 2005;31:1336-1344.

16. Bouhemad B, Liu ZH, Arbelot C, et al. Ultrasound assessment of antibiotic-induced pulmonary reaeration in ventilator-associated pneumonia. Crit Care Med 2010;38:8492.

17. Bouhemad B, Brisson H, Le-Guen M, Arbelot C, Lu Q, Rouby JJ. Bedside ultrasound assessment of positive endexpiratory pressure-induced lung recruitment. Am J Respir Crit Care Med 2011;183:341-347.

18. Yin W, Zou T, Qin Y, et al. Poor lung ultrasound score in shock patients admitted to the ICU is associated with worse outcome. BMC Pulm Med 2019;19:1.

19. Lu X, Arbelot C, Schreiber A, Langeron O, Monsel A, Lu Q. Ultrasound Assessment of Lung Aeration in Subjects Supported by Venovenous Extracorporeal Membrane Oxygenation. Respir Care 2019;64:1478-1487.

20. Caltabeloti F, Monsel A, Arbelot C, et al. Early fluid loading in acute respiratory distress syndrome with septic shock deteriorates lung aeration without impairing arterial oxygenation: a lung ultrasound observational study. Crit Care 2014;18:R91. 